\title{
CONTRIBUTIONS TO THE LOCAL GRAVITATIONAL FIELD FROM BEYOND THE VIRGO SUPERCLUSTER
}

\author{
Amos Yahil \\ State University of New York \\ Stony Brook, NY 11794-2100, USA
}

In the last decade, the inter-relation between the density structure and velocity perturbations in the local Virgo Supercluster (VSC) has provided a major means of determining the cosmological density parameter $\Omega$. The underlying dynamics, the so-called Virgocentric Flow Model (VFM), assumes that the relevant peculiar gravitational field in the VSC is dominated by the monopole term, due to material inside the VSC. Over the past year, however, this premise has been called into question, on both observational and theoretical grounds. It has become clear that the contributions to the local gravitational field from beyond the VSC cannot be neglected. The major new developments are summarized here. A more detailed review is given elsewhere (Yahil 1987).

In order to make the distinction between the internal and external contributions more concrete, imagine the demarcation of the VSC to be a spherical surface. The peculiar gravitational potential inside this volume, due to material outside it, can be written as a multipole expansion:

$$
\phi=\sum \mathrm{a}_{\operatorname{lm}} \mathrm{Y}_{\mathrm{lm}} \mathrm{r}^{\mathrm{l}},
$$

with a corresponding gravitational field

$$
\mathbf{g}=\mathbf{d}+\mathbf{Q} \cdot \mathbf{r}+\ldots
$$

The first term in the expansion is a homogeneous dipole field. (By Newton's theorem there is no monopole term inside the cavity due to material outside it.) The second term is the quadrupolar tidal field, with $Q$ a symmetric and traceless tensor.

In linear perturbation theory the peculiar velocity $\mathbf{u}$ is proportional to $\mathbf{g}$ :

$$
\mathbf{u}=\frac{2}{3} \mathrm{H}_{\mathrm{o}}^{-1} \Omega_{\mathrm{o}}^{-0.4} \mathbf{g}=\mathbf{u}_{\mu}+\sigma \cdot \mathbf{r}+\ldots
$$

The first (dipole) term is the constant bulk velocity of the VSC relative to the Robertson-Walker frame. It can not be measured within the VSC since it cancels for all relative velocities, and therefore needs to be measured relative to the microwave background radiation (MBR), or a sufficiently distant frame of 
galaxies. The quadrupolar tidal velocity field is measurable in principle, and a detection has, in fact, been claimed (Lilje, Yahil, and Jones 1986). Recently, a study of a large sample of elliptical galaxies with $\mathrm{v} \leqslant 6000 \mathrm{~km} \mathrm{~s}^{-1}$ (Dressler et al. 1986) has concluded that the velocity of the Local Group (LG) relative to that frame is different from the one relative to the MBR (Lubin, Epstein, and Smoot 1983; Fixsen, Cheng, and Wilkinson 1983). The new data have not yet been available for independent evaluation, but the plots presented show that the velocity field is turbulent. In fact, for $\mathrm{v} \leqslant 3000 \mathrm{~km} \mathrm{~s}^{-1}$, it resembles the tidal one found by Lilje et al.

A complete dynamical picture, and a means of determining $\Omega_{0}$, is possible only when the kinematic data are coupled to the density structure. The coefficients of the multipole expansion, eq. (1), are given by

$$
\mathrm{a}_{\mathrm{lm}}=\frac{4 \pi}{2 \mathrm{l}+1} \int \rho(\mathbf{r}) \mathrm{r}^{-1-1} \mathrm{Y}_{\mathrm{lm}}^{*}(\Omega) \mathrm{d}^{3} \mathrm{r}
$$

The dipole term is simply the vector sum of the gravitational $\mathrm{r}^{-2}$ attractions of all the structure outside the VSC. The quadrupole term measures the $\mathrm{r}^{-3}$ tidal force.

Complete surveys are needed in order to measure the density of galaxies. The initial RSA catalog was used for the volume inside $|\mathrm{b}|>\mathbf{3 0} 0^{\circ}$ and $\mathrm{v} \lesssim 4000 \mathrm{~km} \mathrm{~s}^{-1}$ (Yahil, Sandage, and Tammann 1980; Yahil 1981). The CfA catalog extends to the greater depth of $8000 \mathrm{~km} \mathrm{~s}^{-1}$, but is confined to $\mathrm{b}>40^{\circ}$, and only limited coverage in the South Galactic Hemisphere (Davis et al. 1980; Davis and Huchra 1982). These studies were unable to find a dipole term in the direction of the MBR. It was therefore concluded that the structure responsible for the bulk velocity of the VSC either lay beyond the observed distances, or in the zone of avoidance, or both.

The advent of the IRAS catalog has made it possible to search further for the illusive density perturbations. The catalog is ideal for this purpose, because it is calibrated homogeneously over almost the entire sky, ranges in distance far beyond the limits of present redshift surveys, and is unaffected by extinction. Two independent teams quickly discovered a dipole anisotropy in the sky distribution of the IRAS galaxies, aligned within the errors with the velocity of the $L G$ relative to the MBR (Yahil, Walker, and Rowan-Robinson 1986; Meiksin and Davis 1986). The interpretation has been that the density structure responsible for the bulk motion of the VSC has been identified.

Most of the IRAS galaxies do not yet have redshifts, but initial surveys have provided the infrared luminosity function (e.g., Lawrence et al. 1986). This can be used to work out the dipole moment of $\mathrm{g}$ (Yahil et al. 1986). When coupled via eq. (3) to the peculiar velocity, assumed to be equal to the velocity of the $L G$ relative to the MBR, the estimate $\Omega \approx 1$ is obtained. This contradicts the previous observational "consensus", based on the VFM, the cosmic virial theorem, and $N$-body calculations, that $\Omega_{0}=0.1-0.2$ (e.g., the review by Yahil 1984).

The theoretical disposition in favor of $\Omega_{0}=1$, now augmented by the observational contradiction, has led to the proposal that galaxy formation is 
more efficient in regions that have higher densities, even though at the epoch of galaxy formation their overdensity may still have been very small (Kaiser 1984; Schaeffer and Silk 1985; Bardeen et al. 1986). As a result of this biased formation process, galaxies are no longer fair tracers of the total mass-energy density. Details of the biasing physics are still sketchy (e.g., Rees 1987).

Biasing is difficult to establish observationally, because mass can only be traced dynamically. It is possible, however, to check whether one type of galaxy has the same density structure as another one. It is well known that the frequencies of early and late type galaxies are functions of density (Dressler 1980), but this has not seemed to bias the determination of the largescale density structure in the VSC (Yahil et al. 1980). It is important to see whether a similar effect might result in different determinations of density from IR (spiral) and optical (spiral and elliptical) galaxies. A comprehensive comparison of the two is not yet possible, because of the lack of redshifts for the IRAS galaxies. However, in the small area of the sky surveyed by Lawrence et al. (1986), $90 \%$ of the IRAS galaxies with $\mathrm{S}_{60} \geq 0.85$ and $\mathrm{v}<3000 \mathrm{~km} \mathrm{~s}^{-1}$ are already included in the CfA catalog. (Of course the CfA catalog also contains many galaxies which are not IRAS galaxies, e.g., elliptical galaxies.) If this is true in all the overlap region between the IRAS and CfA surveys, then their density structures can be compared directly within the limited redshift range $\mathrm{v}<3000 \mathrm{~km} \mathrm{~s}^{-1}$. Such a comparison clearly shows the two density structures to be similar (Yahil 1986). It also shows that the mean cosmological density baseline defined by the IRAS galaxies is higher than that determined in the optical studies. A possible interpretation might be that the existing optical surveys do not extend deep enough, and the volume covered is not a fair sample of the universe. This possibility needs to be examined carefully, both by re-evaluating the existing density determinations, and by extending both the optical and IR redshift surveys.

A full understanding of the density structure of the IRAS galaxies can come only from a complete redshift catalog, which will enable analyses which are not possible with only fluxes and a luminosity function. First, the dipole gravitational field can be determined using individual distances for each galaxy. This will greatly increase the reliability of the result, and minimize biases. Secondly, one can evaluate higher moments of the gravitational field. The quadrupole moment, in particular, should be compared with the quadrupolar tidal velocity field (Lilje et al. 1986). Thirdly, other quantitative descriptions of the density structure around us can be sought, such as correlation functions, hole distribution, and continuous largescale structures. Fourthly, the density structure of IR selected galaxies may be compared with optical galaxies, to obtain some understanding of possible biased galaxy formation. Finally, biased galaxy formation can be tested using the principle of superposition, by determining the effect of introducing density-dependent weighting on the resultant gravitational field (Gunn 1988).

A collaboration (Davis, Huchra, Strauss, Tonry, Yahil) is already underway to obtain a redshift catalog complete to $S_{60} \geq 2 \mathrm{Jy}$ for $|\mathrm{b}|>10^{\circ}$. The flux limit was chosen in order to make the project manageable, with the aim of completing it within two years. The broad luminosity function guarantees that the sample will extend to large distances. The eighty percentile of the redshift 
distribution will be at $10,000 \mathrm{~km} \mathrm{~s}^{-1}$, but there will be adequate sampling even beyond that range. A British team (Efstathiou, Ellis, Frenk, Hewitt, Kaiser, Rowan-Robinson) is undertaking the complimentary approach of sparse sampling the catalog down to $\mathrm{S}_{60} \geq 0.5$. Many of the problems posed above should be answered shortly

This research was supported in part by USDOE grant DE-AC0280ER 10719 at the State University of New York. The hospitality of the Research Institute for Fundamental Physics, Kyoto University, where this work was completed, is gratefully acknowledged.

\section{References}

Bardeen, J.M., Bond, J.R., Kaiser, N., and Szalay, A.S. 1986, Astrophys. J., 304, 15.

Davis, M., Tonry, J., Huchra, J., and Latham, D. 1980, Astrophys. J. (Letters), 238, L113.

Davis, M., and Huchra, J. 1982, Astrophys. J., 254, 437.

Dressler, A. 1980, Astrophys. J., 236, 351.

Dressler, A., Faber, S.M., Burstein, D., Davies, R., Lynden-Bell, D., Terlevich, R., and Wegner, G. 1986, Astrophys. J., submitted.

Fixsen, D.J., Cheng, E.S., and Wilkinson, D.T. 1983, Phys. Rev. Letters, 50, 620.

Gunn, J.E. 1987, in Dark Matter in the Universe, eds. G. Knapp and J. Kormendy (Dordrecht: Reidel).

Kaiser, N. 1984, Astrophys. J. (Letters), 284, L9.

Lilje, P.B., Yahil, A., and Jones, B.J.T. 1986, Astrophys. J., 307, 91.

Lawrence, A., Walker, D., Rowan-Robinson, M., Penston, M., and Leech, K. 1986, Mon. Not. Roy. Astr. Soc., $219,687$.

Lubin, P.M., Epstein, G.L., and Smoot, G.F. 1983, Phys. Rev. Letters, 50, 616.

Meiksin, A., and Davis, M. 1986, Astr. J., 91, 191.

Rees, M. 1987, in Nearly Normal Galaxies: From the Planck Time to the Present, ed. S.M. Faber (Berlin: Springer). in press.

Schaeffer, R., and Silk, J. 1985, Astrophys. J., 292, 319.

Yahil, A. 1981, Ann. N.Y. Acad. Sci., 375, 169. 1984, in The Virgo Cluster of Galaxies, ed O.G. Richter and B. Binggeli (Munich: ESO) pp. 359-373. . 1986, in Galaxy Distances and Deviations from Universal Expansion, eds. B.F. Madore and R.B. Tully (Dordrecht: Reidel) pp. 151-158. . 1987, in Nearly Normal Galaxies: From the Planck Time to the Present, ed. S.M. Faber (Berlin: Springer), in press.

Yahil, A., Walker, D., and Rowan-Robinson, M. 1986, Astrophys. J. (Letters), 301, L1.

Yahil, A., Sandage, A., and Tammann, G.A. 1980, Astrophys. J., 242, 448. 